\title{
Inbreeding depression and outbreeding depression in plants
}

\author{
MIKKEL HEIDE SCHIERUP* \& FREDDY BUGGE CHRISTIANSEN \\ Department of Ecology and Genetics, University of Aarhus, 8000 Aarhus C, Denmark
}

\begin{abstract}
The genetic mechanism underlying an 'optimal outcrossing distance' in plants (sensu Price and Waser) is discussed. Monte Carlo simulations of a population subdivided as a one-dimensional stepping-stone are used to evaluate possible genetic mechanisms. A simple genetic model with two types of unlinked loci, underdominant and partially dominant, with multiplicative effects on fitness, is found to create an 'optimal outcrossing distance' under a wide range of parameter values. The results are compared to, and discussed in relation to a two-locus epistatic model, previously investigated by Campbell and Waser, and found to give very similar results.
\end{abstract}

Keywords: epistasis, mating system evolution, optimal outcrossing, simulation, underdominance.

\section{Introduction}

The phenomenon of an 'optimal outcrossing distance' in plants at the population level has attracted a lot of attention recently (reviewed by Waser, 1993; Waser \& Price, 1994). When plants collected at different geographical distances are crossed artificially, they show a maximum in fitness of offspring of parents collected at intermediate distances. Fitness of the $F_{1}$ plants is determined either in the natural habitat or in a garden or greenhouse. The phenomenon was first observed in Delphinium nelsonii by Price \& Waser (1979) and has now been found in at least 16 different plant species, angiosperms as well as conifers (Parker, 1992; Waser, 1993). The scale of the artificial crossing studies has varied between $75 \mathrm{~m}$ and $32 \mathrm{~km}$, depending on the breeding system of the plant, and the optimal outcrossing distance usually is between 5 and $20 \mathrm{~m}$ for herbs and several hundred meters for conifers (Waser, 1993).

The number of studies is still too limited for an evaluation of how widespread the phenomenon is, and thus its importance in mating system evolution. The existence of an optimal outcrossing distance requires the existence of an optimal genetic similarity (Mitton, 1993) and a correlation between genetic similarity and physical distance of the plants, either through isolation by distance in a continuously distributed population (Wright, 1946) or between semi-isolated patches. Many herbs have

*Correspondence. restricted pollen and seed dispersal (Levin, 1984, 1988; table 9.2 in Waser, 1993) with a paternity pool of only $20-100$ individuals per mother plant (Levin, 1988), and thus small-scale genetic structuring is expected.

Knowledge of the dispersal biology of the plant species of interest is therefore necessary when looking for an optimal outcrossing distance, and some of the failures to observe this phenomenon might be attributed to a paternity pool that is too large compared to the scale of the crosses (Newport, 1989; Broyles \& Wyatt, 1991; Waser \& Price, 1994). Furthermore, large samples are needed to detect reliably the variation in fitness that is characteristic of a situation where an optimal outcrossing distance is observed (Newport, 1989; Waser, 1993).

The phenomenon of optimal outcrossing distance has sufficient generality to discuss its genetic foundation, that is, to investigate genetic models which can lead to disadvantages of both close and far crosses. A combination of inbreeding depression and outbreeding depression is needed, and both these phenomena are poorly understood genetically.

Inbreeding depression has been documented for most investigated plant species, even highly selfing species (Charlesworth \& Charlesworth, 1987). The genetic mechanisms most commonly discussed are the partial dominance hypothesis and the overdominance hypothesis. The partial dominance hypothesis supposes that inbreeding depression is caused by deleterious, partly recessive alleles, and the overdominance hypothesis considers heterozygosity per se as an advantage. Most work supports the partial 
dominance hypothesis (Sprague, 1983; Charlesworth \& Charlesworth, 1987; Fu \& Ritland, 1994). However, Mitton (1993), using a statistical analysis developed by Smouse (1986), claims evidence for overdominance, even though the partial dominance hypothesis predicts widespread occurrence of associative overdominance in a finite population (Frydenberg, 1964; Kimura \& Ohta, 1971; Christiansen, 1990). A third, rarely discussed, explanation is that epistatic interactions can cause inbreeding depression (Lynch, 1991). Campbell \& Waser (1987) confirmed this as a possible mechanism by the use of computer simulations.

Another debated topic is whether inbreeding depression is caused by few or many loci with small or large effects, respectively. Highly deleterious recessive alleles (as, e.g. recessive lethals) are common in outcrossing species, but the persistence of inbreeding depression in highly selfing species suggests that many loci of small effect is the most plausible explanation of inbreeding depression in general (Charlesworth \& Charlesworth, 1987; Ågren \& Schemske, 1993; Wolfe, 1993).

Outbreeding depression is the population level counterpart to mechanisms separating species or subspecies. Discussions of the causes have been divided into an 'ecological' mechanism (Price \& Waser, 1979; Campbell \& Waser, 1987; Waser, 1993) and a 'genetic' mechanism of epistatic interactions between multilocus gene complexes (Price \& Waser, 1979; Lynch, 1991). The ecological mechanism assumes that subpopulations are differentiated by the adaptation to different environments at microsites. Crosses between sites are then expected to yield maladapted offspring. With this mechanism, the driving force is a combination of local selection, drift and gene flow, and the hypothesis can be tested through reciprocal transplants of the progeny of artificial crosses. Waser (1993) reviews the accumulated evidence and concludes that some of the cases of outbreeding depression are attributable to this mechanism (see also Waser \& Price, 1989, 1993, 1994). The genetic mechanism assumes an identical environment in all populations and that restricted gene flow between and drift within populations by chance create coadapted gene complexes by epistatic interactions (Moll et al., 1965; Campbell \& Waser, 1987; Mitton, 1993). These complexes are broken up by crosses between sites, but under these genetic models, the major effect will occur after segregation in the $F_{2}$ generation. Because of a lack of detectable environmental heterogeneity, Svensson (1988) and Parker (1992) claim that their evidence for outbreeding depression must be ascribed to a genetic mechanism. A second possibility for genetic outbreeding depression is underdominance at a number of loci (Ritland \& Ganders, 1987; Lynch, 1991). This genetic mechanism has full effect in the $F_{1}$ generation, and it results from intralocus interaction rather than epistasis. We study this mechanism and investigate its possibilities in greater detail.

\section{The models}

We consider a subdivided population where migration is structured, as in the one-dimensional stepping-stone model of Kimura \& Weiss (1964). This model is easier to study than the two-dimensional model and it yields qualitatively similar results. Migration between adjacent demes occurs as gametes and at a constant rate with the exchange of a fraction $m / 2$ per generation. To avoid edge effects in the model, the subpopulations are arranged in a circle (Kimura \& Maruyama, 1971). The circle consists of $S$ populations with population size $N$. Monte Carlo simulations were performed with a total population of 1600 (or 3200) individuals, divided into either $S=80$ subpopulations with $N=20$ (40) individuals in each, or $S=160$ subpopulations with $N=10$ individuals in each. Gametic fusion occurs after migration with a random amount of selfing (selfing rate $=1 /(2 N)$ in an isolated population). Individuals vary in their fitness, and so they are chosen to contribute gametes by random drawing with their presence in the population weighted by the fitness.

Two different genetic models were investigated, a model including underdominant loci and a model including the two-locus epistatic model of Campbell \& Waser (1987). The genetic models both include a number of loci, all unlinked and with two alleles each.

\section{The underdominance model}

Table 1 presents this model, which consists of two types of loci. First, the table presents partially dominant loci which are responsible for inbreeding

Table 1 Genotypic fitnesses under the underdominance model

\begin{tabular}{lccc}
\hline & $A_{1} A_{1}$ & $A_{1} A_{2}$ & $A_{2} A_{2}$ \\
Partial dominance & 1 & $1-h s$ & $1-s$ \\
& $B_{1} B_{1}$ & $B_{1} B_{2}$ & $B_{2} B_{2}$ \\
Underdominance & 1 & $1-t$ & 1 \\
\hline
\end{tabular}

(c) The Genetical Society of Great Britain, Heredity, 77, 461-468. 
depression under the partial dominance hypothesis. The selection coefficient against the deleterious homozygote is denoted $s>0$, and $h$ is the dominance coefficient. We assume positive dominance of the normal homozygote, i.e. $0 \leq h<1 / 2$. Variation at this type of locus is introduced by mutations at a rate of $10^{-3}$. Secondly, the table presents the underdominant loci that are expected to cause outbreeding depression under the underdominance model. For these, $t>0$ is the selection coefficient against the heterozygote. With no mutation, both monomorphic states are stable equilibria. Variation at the underdominant loci is introduced initially in each Monte Carlo simulation and maintained by a mutation rate of $10^{-3}$. Total individual fitness was calculated by combining fitness coefficients of loci multiplicatively. The number of loci used in the simulations of the underdominance model was 100 or 200 for the partially dominant loci, and 20 for the underdominant loci.

\section{The epistasis model}

This model (Table 2) is built from the two-locus epistatic model of Campbell \& Waser (1987) which is a version of the symmetric viability model (Lewontin \& Kojima, 1960). The fitness coefficients for each combination of genotypes at two interacting loci are shown in the table, where $\alpha$ is the selection coefficient against double homozygotes $(\alpha>0)$, and $\varepsilon$ is the selection coefficient against double heterozygotes $(\varepsilon>0)$. With no mutation, four stable equilibria exist for $\alpha<\varepsilon$, and at these equilibria, one of the interacting loci is fixed at a monomorphic state and the other is polymorphic with even allele frequencies. For $\alpha>\varepsilon$, only one stable polymorphic equilibrium exists where even allele frequencies and linkage equilibrium prevail. Campbell \& Waser (1987) used this two-locus model with quite high selection coefficients in their simulations. We use the two-locus model as an atom in a multilocus model, and total individual fitness was calculated by combining fitness coefficients of the pairs of interacting loci multiplicatively. Variation was introduced by mutation at a rate of $10^{-3}$ at each locus. The

Table 2 Genotypic fitnesses under the epistasis model

\begin{tabular}{cccc}
\hline & $A_{1} A_{1}$ & $A_{1} A_{2}$ & $A_{2} A_{2}$ \\
$B_{1} B_{1}$ & $1-\alpha$ & 1 & $1-\alpha$ \\
$B_{1} B_{2}$ & 1 & $1-\varepsilon$ & 1 \\
$B_{2} B_{2}$ & $1-\alpha$ & 1 & $1-\alpha$ \\
\hline
\end{tabular}

number of loci used in the simulations of the epistasis model was again 100 and 200 , distributed as 50 or 100 pairs of interacting loci.

In each simulation run, the population was allowed to evolve until an approximate mutationselection-drift equilibrium was reached, typically after 2000 generations. Crosses were then performed by randomly choosing gametes from subpopulations separated by different distances (from 0 to $S / 2$ ). Subsequently, the average fitness of the offspring from each cross was calculated by choosing 100 random crosses from each distance class. For all sets of parameters, five replicate simulations were performed to get an evaluation of the variance in average fitness.

The simulation program was written in $\mathrm{C}++$ and run on a HP-UNIX workstation. Random numbers were generated using the RANLIB 1.1 random generator.

\section{Results}

\section{The underdominance model}

Figures 1 and 2 show the results for two distinct sets of parameters. The shapes of the curves are well defined, with small standard errors. Therefore, the graphs in general allow the estimation of 'the optimal outcrossing distance', and we may present

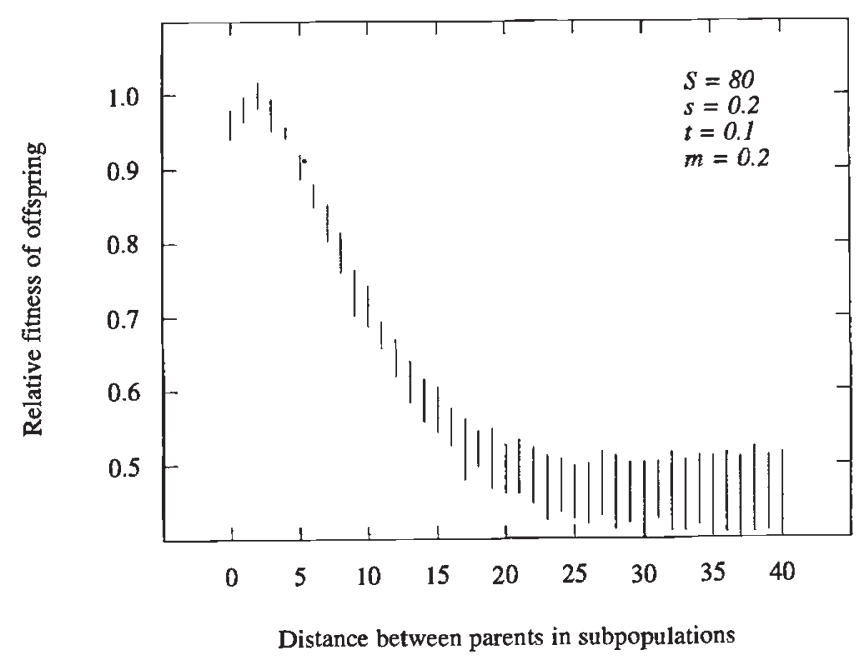

Fig. 1 The relation between the distance between parents and the offspring fitness under the underdominance model. There are 80 subpopulations with 20 individuals each. Migration is 20 per cent. The number of underdominant loci is 20 with $t=0.1$ and there are 100 partially dominant loci with $s=0.2$. Standard error bars are based on five replicate runs. Fitnesses are shown relative to the optimum. 
the results of all runs as in Tables 3 and 4. An optimal offspring fitness was found at an intermediate crossing distance for all tested parameter values.

Some patterns are suggested from the results in Tables 3 and 4. A decrease in the number of individuals per subpopulation, or an increase in the migration rate, increases the optimal outcrossing distance. Comparing inbreeding caused by either 100 loci with $s=0.2$, or 200 loci with $s=0.1$ (the same mutational

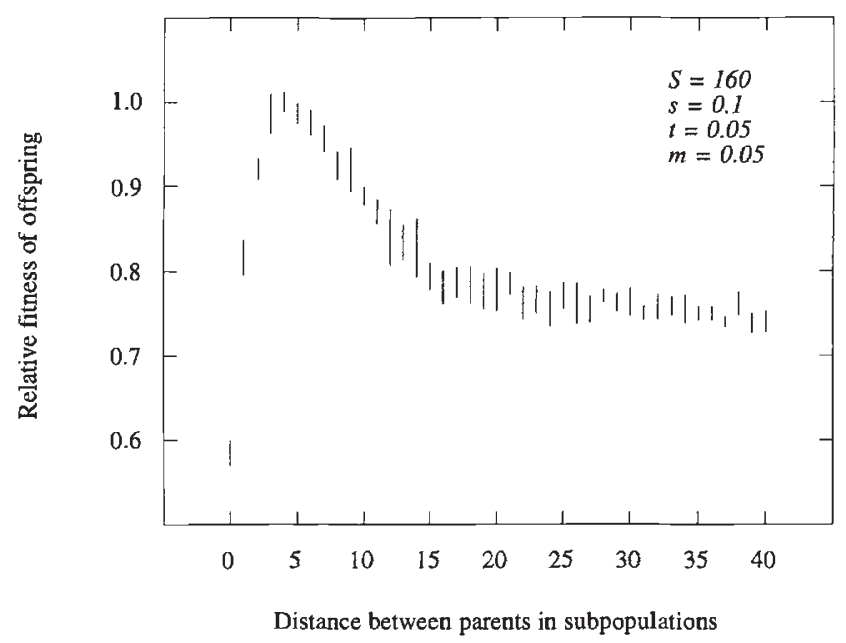

Fig. 2 As Fig. 1, but with a different set of parameter values. There are 160 subpopulations with 10 individuals each. Migration is 5 per cent. The number of underdominant loci is 20 with $t=0.05$ and there are 200 partially dominant loci with $s=0.1$. load), less inbreeding depression at equilibrium is observed in the first case. As expected, this lowers the optimal outcrossing distance when the outbreeding depression is kept constant. Similarly, when outbreeding depression is increased by increasing $t$, the optimal outbreeding distance is smaller.

Inbreeding depression is generally larger when the 1600 individuals are separated in subpopulations of size 10 than subpopulations of size 20 . This is to be expected, because drift is more powerful with only 10 individuals per subpopulation, and more deleterious recessive alleles can increase in frequency in spite of selective disadvantage.

\section{The epistasis model}

The results of the runs with the two-locus epistasis model, with $\alpha<\varepsilon$, are shown in Table 5. They show that it is possible to create an 'optimal outcrossing distance' with this model too, and the trends, when changing the parameters, are very similar to the trends observed with the underdominance model. Some runs were also performed with $\alpha>\varepsilon$ (data not shown), but in this case outbreeding depression was not observed (in concordance with Campbell \& Waser, 1987, figs 3 and 4).

\section{Discussion}

Inbreeding depression and outbreeding depression may occur simultaneously in simple genetic models.

Table 3 The underdominance model. Results are based on five replicate simulations. Outbreeding depression is caused by 20 underdominant loci, and inbreeding depression is caused by 100 loci with $s=0.2$. Fitnesses of near and wide crosses are given relative to the fitness at the optimal distance

\begin{tabular}{|c|c|c|c|c|c|c|}
\hline $\begin{array}{l}\text { No. of } \\
\text { subpops }\end{array}$ & $\begin{array}{l}\text { Ind. per } \\
\text { subpop } \\
(N)\end{array}$ & $t$ & $\begin{array}{l}\text { Migration } \\
\quad(m)\end{array}$ & $\begin{array}{c}\text { Optimal } \\
\text { distance } \\
\text { (no. of subpops) }\end{array}$ & $\begin{array}{l}\text { Fitness of } \\
\text { near cross } \\
\text { (0 subpops) }\end{array}$ & $\begin{array}{c}\text { Fitness of } \\
\text { wide cross } \\
\text { (40 subpops) }\end{array}$ \\
\hline 80 & 20 & 0.10 & 0.05 & 1 & 0.90 & 0.46 \\
\hline 80 & 20 & 0.10 & 0.20 & 2 & 0.96 & 0.49 \\
\hline 80 & 20 & 0.05 & 0.05 & 2 & 0.89 & 0.73 \\
\hline 80 & 20 & 0.05 & 0.20 & 4.5 & 0.94 & 0.77 \\
\hline 80 & 40 & 0.10 & 0.05 & 1 & 0.99 & 0.43 \\
\hline 80 & 40 & 0.10 & 0.20 & 1 & 0.96 & 0.50 \\
\hline 80 & 40 & 0.05 & 0.05 & 1 & 0.93 & 0.69 \\
\hline 80 & 40 & 0.05 & 0.20 & 2 & 0.95 & 0.73 \\
\hline 160 & 10 & 0.10 & 0.05 & 1.5 & 0.90 & 0.46 \\
\hline 160 & 10 & 0.10 & 0.20 & 2.5 & 0.92 & 0.55 \\
\hline 160 & 10 & 0.10 & 0.40 & 3 & 0.94 & 0.55 \\
\hline 160 & 10 & 0.05 & 0.05 & 3 & 0.82 & 0.69 \\
\hline 160 & 10 & 0.05 & 0.20 & 3.5 & 0.87 & 0.69 \\
\hline 160 & 10 & 0.05 & 0.40 & 5 & 0.91 & 0.72 \\
\hline
\end{tabular}


Table 4 The underdominance model. As Table 3 but with inbreeding depression caused by 200 loci with $s=0.1$

\begin{tabular}{lcccccc}
\hline $\begin{array}{l}\text { No. of } \\
\text { subpops }\end{array}$ & $\begin{array}{c}\text { Ind. per } \\
\text { subpop } \\
(N)\end{array}$ & $t$ & $\begin{array}{c}\text { Migration } \\
(\mathrm{m})\end{array}$ & $\begin{array}{c}\text { Optimal } \\
\text { distance } \\
\text { (no. of subpops) }\end{array}$ & $\begin{array}{c}\text { Fitness of } \\
\text { near cross } \\
\text { (0 subpops) }\end{array}$ & $\begin{array}{c}\text { Fitness of } \\
\text { wide cross } \\
\text { (40 subpops) }\end{array}$ \\
\hline 80 & 20 & 0.10 & 0.05 & 2 & 0.81 & 0.47 \\
80 & 20 & 0.10 & 0.20 & 4 & 0.92 & 0.48 \\
80 & 20 & 0.05 & 0.05 & 3 & 0.78 & 0.75 \\
80 & 20 & 0.05 & 0.20 & 5 & 0.86 & 0.74 \\
80 & 40 & 0.10 & 0.05 & 1.5 & 0.98 & 0.47 \\
80 & 40 & 0.10 & 0.20 & 2 & 0.87 & 0.70 \\
80 & 40 & 0.05 & 0.05 & 3 & 0.95 & 0.75 \\
80 & 40 & 0.05 & 0.20 & 4 & 0.79 & 0.57 \\
160 & 10 & 0.10 & 0.05 & 3 & 0.85 & 0.58 \\
160 & 10 & 0.10 & 0.20 & 5 & 0.58 & 0.75 \\
160 & 10 & 0.10 & 0.40 & 7 & 0.72 & 0.79 \\
160 & 10 & 0.05 & 0.05 & 4 & 6 & 0.83 \\
160 & 10 & 0.05 & 0.20 & 6 & & 0.87 \\
160 & 10 & 0.05 & 0.40 & 9 & & \\
\hline
\end{tabular}

Table 5 The epistasis model. Results are based on five replicate simulations of a population with 80 subpopulations with 20 individuals each. Fitnesses of near and wide crosses are given relative to the fitness at the optimal distance

\begin{tabular}{lcccccc}
\hline $\begin{array}{l}\text { Number of } \\
\text { pairs of loci }\end{array}$ & $\alpha$ & $\varepsilon$ & $\begin{array}{c}\text { Migration } \\
(m)\end{array}$ & $\begin{array}{c}\text { Optimal } \\
\text { distance }\end{array}$ & $\begin{array}{c}\text { Fitness of } \\
\text { near cross } \\
(0 \text { subpops })\end{array}$ & $\begin{array}{c}\text { Fitness of } \\
\text { wide cross } \\
(40 \text { subpops })\end{array}$ \\
\hline 50 & 0.05 & 0.10 & 0.05 & 4 & 0.83 & 0.75 \\
50 & 0.05 & 0.10 & 0.20 & 5 & 0.89 & 0.73 \\
50 & 0.05 & 0.10 & 0.40 & 6 & 0.95 & 0.80 \\
50 & 0.10 & 0.20 & 0.05 & 1.5 & 0.73 & 0.45 \\
50 & 0.10 & 0.20 & 0.20 & 3 & 0.85 & 0.55 \\
50 & 0.10 & 0.20 & 0.40 & 4 & 0.95 & 0.72 \\
100 & 0.025 & 0.05 & 0.05 & 3 & 0.83 & 0.80 \\
100 & 0.025 & 0.05 & 0.40 & 6 & 0.96 & 0.84 \\
100 & 0.05 & 0.10 & 0.05 & 2 & 0.67 & 0.58 \\
100 & 0.05 & 0.10 & 0.40 & 3 & 0.91 & 0.56 \\
\hline
\end{tabular}

Campbell \& Waser (1987) showed this phenomenon in a simple two-locus model and considered it to be based on epistatic interactions. It seems, however, that it is not epistatic interactions per se that cause outbreeding depression, but rather the occurrence of simultaneously stable distinct equilibria. Campbell \& Waser's model produces outbreeding depression only when competing equilibria exist in an isolated population. This occurs when the double heterozygote for a pair of interacting loci has a lower fitness than the corresponding double homozygote $(\alpha<\varepsilon)$. The competing equilibria in the two-locus model are all at local maxima in mean fitness in an isolated population, and a low amount of immigration is only expected to perturb the population slightly from these equilibria (Christiansen \& Feldman, 1975), whereas offspring from crosses between populations at different equilibria will show an excess of the disfavoured double heterozygotes. If the opposite is the case, and the double heterozygote has a higher fitness than the corresponding double homozygote $(\alpha>\varepsilon)$, then an isolated population has only one stable equilibrium which happens to be at an optimum of the average fitness. Divergence among the populations is therefore not expected and outbreeding depression is absent.

The simplest model of outbreeding depression must be a one-locus model that exhibits competing 
equilibria stabilized at optima of the average fitness. The results show that it is indeed possible to generate the phenomenon of an 'optimal outcrossing distance' with such a simple genetic model. In our version the model assumes two types of independent loci, with underdominance in fitness and with partial dominance, respectively. This simple model yields fitness values of $F_{1}$ crosses which are comparable with the published results (Waser, 1993). Simultaneous occurrence of inbreeding depression and outbreeding depression is expected in a model with only underdominant loci, because this model has dynamical properties parallel to those of the two-locus model. The inclusion of the partially dominant loci, however, provides a more realistic model of inbreeding depression.

The reason for the occurrence of an 'optimal outcrossing distance' may be that, through drift and selection, the two types of loci get a different patch structure, as formulated verbally by Ritland \& Ganders (1987). For the partially dominant loci, deleterious alleles causing inbreeding depression may reach local high frequencies resulting from random fluctuations caused by the small size of subpopulations. The extent of the local 'patch' of high frequency for a particular deleterious allele increases with the amount of migration, and the amount of local differentiation at the partially dominant loci weakens with more migration. The underdominant loci causing outbreeding depression may be fixed, or almost fixed, for different alleles in different parts of the total population (Karlin \& McGregor, 1972). The patch size for high frequency of a particular allele is highly dependent on random events at the start of the simulation, and less influenced by local migration. The optimal outcrossing distance is then found at a distance which is larger than the patch size for recessive deleterious alleles at the partially dominant loci, and smaller than the patch size for alleles at the underdominant loci, if a difference in the patch size exists.

The simulations rely on a high mutation rate, $u=0.001$. This has been chosen for computational reasons to reduce the number of loci, and to decrease the time required to reach equilibrium. Simulations with the number of loci increased and the mutation rate decreased yielded qualitatively identical results.

Underdominant loci have been identified in only a few cases (Fu \& Ritland, 1994). The reason may be that underdominant loci are indeed rare, but they also may be difficult to see, when selection against the heterozygote is weak. With weak selection, the locus behaves almost as a neutral locus, and the heterozygote deficit will be difficult to detect. Epistatic interactions within populations in the sense of Campbell \& Waser's model are even less documented, and preference should in our opinion be given to the simpler one-locus model.

The two-locus epistatic model and the single-locus model are both used as atoms of a multilocus model. Comparing the two selection models, Campbell \& Waser's symmetric viability model shows negative epistasis whether measured on a linear or a multiplicative scale (Christiansen, 1988), but the multiplicative combination of the atoms is an operation emphasizing positive epistasis (the linear epistasis parameters for two, four, six and eight loci are $-(\alpha+\varepsilon),(\alpha+\varepsilon)^{2},-(\alpha+\varepsilon)^{3}$ and $(\alpha+\varepsilon)^{4}$, respectively). On the contrary, the proposed underdominance model shows positive epistasis on a linear scale and zero epistasis on a multiplicative scale. The two kinds of models, however, give parallel results and share the property of exhibiting competing stable equilibria at local fitness maxima. Competing stable equilibria are ubiquitous in multilocus models. The two-locus symmetric viability model of Lewontin \& Kojima (1960) may exhibit competing equilibria in a range of situations. Our underdominance model in a two-locus version corresponds to $1-\alpha=1 /(1-\varepsilon)$ and $\varepsilon>0$ (Table 2) and provides an example of the situations where all monomorphic equilibria are stable. The epistasis model of Campbell \& Waser is an example of four competing equilibria with one-locus polymorphism. Both these examples assume that the double heterozygotes have inferior fitness $(\alpha<\varepsilon)$, but even when double heterozygotes have a higher fitness than single heterozygotes $(\varepsilon<0)$, and single heterozygotes have higher fitness than homozygotes $(\alpha>0)$, competing equilibria may exist (Lewontin \& Kojima, 1960). These equilibria, called high complementarity equilibria, may exist for closely linked loci, and they are characterized by polymorphism at both loci and linkage disequilibrium. The high complementarity equilibria may cause differentiation between semiisolated populations (Christiansen \& Feldman, 1975). The high complementarity equilibria are not at local fitness maxima, but even then, they may cause outbreeding depression because they are separated by a trough in average fitness, and so immigration perturbs the population towards a state with lower fitness. This possibility of 'overdominant' systems with the potential of producing outbreeding depression seems to proliferate in multilocus models of partially linked loci (Feldman et al., 1974; Christiansen, 1988).

The plants are able to realize the optimal distance 
through mating only in the few cases where the optimal outcrossing distance is one (Table 3). This is in agreement with the empirical findings that the optimal distance is often several neighbourhoods wide (Waser, 1993). This, however, does not imply that the phenomenon is unimportant in mating system evolution. The simulations show that when migration is increased, the optimal outcrossing distance increases. Therefore, even when increased gene flow is selected for, the plants will not be able to reach the optimum crossing distance and there may possibly be a runaway selection for increasing gene flow, until other factors become important.

\section{Acknowledgements}

The study was supported by grants from the Danish Natural Sciences Research Council (grant no. 9400065 to M.H.S and grant no. 11-9639-1 to F.B.C), and a grant to F.B.C. from the Development Programme for Research in Biotechnology (grant no. 5.18.2023).

\section{References}

ÅGREN, J. AND SCHEMSKE, D. W. 1993. Outcrossing rate and inbreeding depression in two annual monoecious herbs, Begonia hirsuta and B. semiovata. Evolution, 47, 125-135.

BROYlES, S. B. AND WYATT, R. 1991. Effective pollen dispersal in a natural population of Asclepias exaltata: the influence of pollinator behavior, genetic similarity, and mating success. Am. Nat., 138, 1239-1249.

CAMPBELL, D. R. AND WASER, N. M. 1987. The evolution of plant mating systems: multilocus simulations of pollen dispersal. Am. Nat., 129, 593-609.

CHARLESWORTH, D. AND CHARLESWORTH, B. 1987. Inbreeding depression and its evolutionary consequences. Ann. Rev. Ecol. Syst., 18, 237-268.

CHRISTIANSEN, F. B. 1988. Epistasis in the multiple locus viability model. J. Math. Biol., 26, 595-618.

CHRISTIANSEN, F. B. 1990. Population consequences of genetic design in sexually reproducing organisms. In: Mooney, H. and Bernardi, G. (eds) Genetically Designed Organisms in the Environment, pp. 43-55. John Wiley \& Sons, London.

CHRISTIANSEN, F. B. AND FELDMAN, M. W. 1975. Subdivided populations: a review of the one- and two-locus theory. Theor. Pop. Biol., 7, 13-38.

FELDMAN, M. W., FRANKLIN, F. R. AND THOMSON, G. J. 1974. Selection in complex genetic systems. 1. The symmetric equilibria of the three-locus viability model. Genetics, 76, 135-162.

FRYDENBERG, O. 1964. Long-term instability of an ebony polymorphism in artificial populations of Drosophila melanogaster. Hereditas, 51, 198-206.
FU, Y.-B. AND RITLAND, K. 1994. Evidence for the partial dominance of viability genes contributing to inbreeding depression in Mimulus guttatus. Genetics, 136, 323-331.

KARlin, S. AND McGregor, J. 1972. Polymorphisms for genetic and ecological systems with weak coupling. Theor. Pop. Biol., 3, 210-238.

KIMURA, M. AND MARUYAMA, T. 1971. Pattern of neutral polymorphism in a geographically structured population. Genet. Res. , 18, 125-131.

kimura, M. AND ohta, T. 1971. Theoretical Aspects of Population Genetics. Princeton University Press, Princeton, NJ.

KIMURA, M. AND WeISS, G. H. 1964. The stepping stone model of population structure and the decrease of genetic correlation with distance. Genetics, 49, 561-576.

LEVIN, D. A. 1984. Inbreeding depression and proximitydependent crossing success in Phlox drummondii. Evolution, 36, 116-127.

LEVIN, D. A. 1988. The paternity pools of plants. Am. Nat., 132, 309-317.

LEWONTIN, R. C. AND KOJIMA, K. 1960. The evolutionary dynamics of complex polymorphisms. Evolution, 14, 458-472.

LYNCH, M. 1991. The genetic interpretation of inbreeding depression and outbreeding depression. Evolution, 45, 622-629.

MITTON, J. B. 1993. Theory and data pertinent to the relationship between heterozygosity and fitness. In: Thornhill, N. W. (ed.) The Natural History of Inbreeding and Outbreeding, pp. 17-41. The University of Chicago Press, Chicago.

MOLL, R. H., LONNQUIST, J. H., VELEZFOURTUNO, J. AND JOHNSON, E. C. 1965. The relationship of heterosis and genetic divergence in maize. Genetics, 52, 139-144.

NEWPORT, M. E. A. 1989. A test for proximity-dependent outcrossing in the alpine skypilot, Polemonium viscosum. Evolution, 43, 1110-1113.

PARKER, M. A. 1992. Outbreeding depression in a selfing annual. Evolution, 46, 837-841.

PRICE, M. V. AND WASER, N. M. 1979. Pollen dispersal and optimal outcrossing in Delphinium nelsonii. Nature, 277, 294-297.

RITLAND, K. AND GANDERS, F. R. 1987. Crossability of Mimulus guttatus in relation to components of gene fixation. Evolution, 41, 772-786.

SMOUSE, P. E. 1986. The fitness consequences of multiplelocus heterozygosity under the multiplicative overdominance and inbreeding depression models. Evolution, 40, 946-957.

SPRAGUE, G. F. 1983. Heterosis in maize: theory and practice. In: Frankel, R. (ed.) Heterosis: Reappraisal of Theory and Practice, pp. 22-35. Springer Verlag, Berlin.

SVENSSON, L. 1988. Inbreeding, crossing and variation in stamen number in Scleranthus annuus (Caryophyllaceae), a selfing annual. Evol. Trends Plants, 2, 31-37.

WASER, N. M. 1993. Population structure, optimal outbreeding, and assortative mating in angiosperms. In: Thornhill, N. W. (ed.) The Natural History of Inbreeding and Outbreeding, pp. 173-199. The University of 
Chicago Press, Chicago.

WASER, N. M. AND PRICE, M. v. 1989. Optimal outcrossing in Ipomopsis aggregata: seed set and offspring fitness. Evolution, 43, 1097-1109.

WASER, N. M. AND PRICE, M. V. 1993. Crossing distance effects on prezygotic performances in plants: an argument for female choice. Oikos, 68, 303-308.

WASER, N. M. AND PRICE, M. V. 1994. Crossing-distance effects in Delphinium nelsonit: outbreeding and inbreeding depression in progeny fitness. Evolution, 48, $842-852$.

WOLFE, L. M. 1993. Inbreeding depression in Hydrophyllum appendiculatum: role of maternal effects, crowding, and parental mating history. Evolution, 47, 374-386.

WRIGHT, s. 1946. Isolation by distance under diverse systems of mating. Genetics, 31, 39-59. 